\title{
A CARICATURA GRÁFICA E 0 ETHOS POPULAR EM POMPÉIA
}

\author{
PEDRO PAULO ABREU FUNARI \\ Universidade Estadual Paulista
}

\begin{abstract}
Resumo: A partir do popular e do satrico, busca-se a definiçăo do ethos popular em Pompeia. Parte-se de uma análise da cultura popular na sociedade antiga, ressaltando-se a sátira e a caricatura como formas expressivas especificamente populares. O simbolismo estil/stico presente no grafismo parietal pompeiano é estudado em contraposiçăo ds expressóes eruditas (carmina figurata). Caracteriza-se o sistema sodcio-semiótico das caricaturas pompeianas chegando-se a especificidade do ethos popular.
\end{abstract}

Palavras-chave: Caricatura; cultura popular; Pompeia; cultura romana; grafismo.

\section{DO POPULAR AO SATÍRICO: EM BUSCA DO ETHOS POPULAR}

Desde suas origens, no século dezenove, os estudos da cultura popular têm sido marcados por discussóes epistemológicas sobre a especificidade de seu objeto de estudo. A "sabedoria popular" ou folclore, como passou a ser chamada a partir dos anos 1840 , foi identificada, algumas vezes com a tradição oral (Sebillot, 1913, p.6), principalmente produzida por camponeses iletrados ignorantes das regras dos padrōes de elite ou oficiais (Est populaire tout ce qui n'est pas officiel, definiria Marcel Mauss). Talvez o melhor exemplo deste ponto de vista encontre-se na ênfase dada a composições como as mnemonias:

Um, dois, feijāo com arroz;

Trés, quatro, feijão no prato;

Cinco, seis, feijăo português;

Sete, oito, feijão com biscoito;

Nove, dez, feijăo com pastêis.

Apenas neste século iria se desenvolveruma crftica a esta abordagem e os escritos de Croce, particularmente sua "Poesia popular e poesia artistica", datada do final dos anos 1920, iriam por em questão algumas das caracterlsticas comumente aceitas nos pioneiros estudos do Folclore (Croce, s/d, p. 342), como sua contraposição entre Volkslied e Kunstlied. Mikhail Bakhtine (Bakhtine, 1970, pp. 19, 21, 25 et passim) propunha que a cultura popular fosse caracterizada por brincadeiras, ritos cômicos (narodnii smekh) assim como por insultos de cunho apotropaico (cf. Burke 1989a, p. 103). Contudo, somente mais tarde a "História dos de baixo" (History from below, Hill, 1989, p. 12) começaria a produzir monografias sobre a cultura popular medieval (Rosenberg, 1980), moderna (Burke, 1989; Hoggart, 1986) e contemporânea (Golby \& Pardue, 1984) e suas implicaçōes teórico-metodológicas (cf. Woolen, 1991, p. 72). Uma "História cultural dos pobres" (Howkins, 1990, p. 120) implica no reconhecimento do efeito potencialmente subversivo e revolucionário da cultura popular (Browne, 1989, p. 14) e, ainda, da pluralidade tanto das culturas populares como eruditas (Burke, 1989b, p. 20-1) e de sua interdependência. Entretanto, concordo com Carlo Guinsburg (Guinsburg, 1986, p. 108) que "a bi- 
partição entre cultura popular e erudita é mais útil do que um modelo holístico" que não captaria corretamente a especificidade das expressōes populares considerando-as como derivadas da "cultura dominante" (Trigger, 1989, p. 786).

Parece-me, contudo, que persiste, ainda, uma certa incerteza sobre a caracterização da cultura popular. A definição negativa como culturas que não são da elite (Burke, 1989, p. 15) é o suficientemente boa em termos sociológicos mas seria possivel definfla ontologicamente? As instigantes proposições metafisicas de Croce (Croce, s/d, p. 345) a respeito merecem ser citadas: "a poesia (ou a cultura) popular exprime movimentos da alma que não têm atrás de si, como precedentes imediatos, grandes elaborações do pensamento e da paixāo; descreve sentimentos simples em formas correspondentes simples. A alta poesia (ou cultura) move e desperta grandes massas de recordaçōes, experiências, pensamentos, múltiplos sentimentos com diferentes graus de sutileza; a poesia (ou cultura) popular não se alarga por tão amplos caminhos para chegar ao sentido, mas o atinge de maneira breve e rápida". A oposição crociana entre a experiência, pensamento e sentimento da elite, com diferentes graus expressivos e o caráter direto do popular talvez não seja completamente plauslvel mas, ao menos, sua interpretação levanta algumas questões importantes sobre suas diferenças ontológicas. Como as culturas de classe são historicamente determinadas, qualquer definição ontológica depende da compreensão dos contextos históricos e sociais especlficos através de uma análise microscópica (Nicolet, 1988, p. 40). Os desenhos parietais pompeianos adequam-se, perfeitamente, a tal estudo, como espero demonstrar neste artigo. Antes disso, entretanto, devemos considerar como a cultura popular tem sido vistó no contexto da sociedade romana.

\section{A CULTURA POPULAR E A SOCIEDADE ANTIGA}

Quando Mikhail Rostovtzeff (Rostovtzeff, 1911, p. 141) escreveu seu exaustivo artigo sobre a paisagem arquitetural romana e helenistica, pareceu-Ihe absolutamente natural (Brunt, 1983 , p. 95) citar Vitrúvio $(7,5)$, em sua famosa descrição da pintura parietal das casas ricas, sem levar em conta seu viés erudito (cf. Hahn, 1991, p. 364; Bulford, 1972, p. 25): pinguntur enim portus promuntoria litora flumina fontes euripi fana luci montes pecora pastores. As habitaçōes populares (Hobson, 1985; Scobie, 1986) não entravam em seu discurso. R. Bianchi Bandinelli (Bandinelli, 1970, p. 64), ainda que não tratasse, explicitamente, da expressão "popular", propós uma análise de classe que distingüisse as tendências "senatorial" e "plebéia" (Bandinelli, 1981, p. 45; anteriormente, havia preferido denominar esta última de "corrente popular", Bandinelli, 1961, pp. 231-2). Este "realismo popular tosco" (Brendel, 1979, p. 9) tratava, contudo, de rêterenciais tardios, de ambiente provincial, de classe média (Rodenwaldt, 1939, p. 547) e não da expressão de cunho efetivamente popular. Já no início dos anos $1930 \mathrm{E}$. Lissberger (Lissberger, 1934), em sua palestra inaugural em Tubingen, ressaltava que as evidências epigráficas sugeriam um alto nivel de alfabetização e criatividade entre a população humilde (cf. Guillemin, 1935, p. 404).

A despeito do pessimismo de alguns estudiosos a respeito de nossa acessibilidade às evidéncias populares (cf. McMullen, 1990, p. 186) ou de sua caracterização como grosseira e vulgar (Cebe, 1966, p. 372, referindo-se a grafites), tem-se tido uma crescente consciéncia de que visões comumente aceitas, como o chamado "desprezo dos antigos por atividades manuais", não podem ser aplicadas às visões de mundo populares (cf. MacMullen, 1974, pp. 120; 202, com discussăo da bibliografia anterior; Wood, 1989, p. 137 et passim). Um alto grau de alfabetização entre a população romana, graças aos estudos de caso das evidẻncias epigráficas (Gichon, 1983, p. 585; Funari, 1989), vem a reforçar a impressão que, embora houvesse diferentes classes (De Martino, 1988, p. 223) e culturas (Mattews, 1990, p. 339) populares, a Romanização (Orsted, 1985, p. 11) acabaria por levar à constituição de uma koiné popular, abrangendo a maioria dos escravos e dos trabalhadores pobres (Harris, 1988, p. 603). Os grafites pompeianos permitem-nos, de maneira única, observar elementos de classe presentes no 
discurso e visão populares (cf. Dlaz, 1990, p. 499). Neste artigo, tratarei somente das caricaturas, deixando, assim, de lado as mensagens verbais, por dois motivos: em primeiro lugar, para tentar abordar o ethos popular através da representação gráfica, particularmente, do exagero; em segundo lugar, para limitar o universo de análise, neste estágio da pesquisa, a um corpus não tão amplo.

\section{SÁTIRA, CARICATURA: O SIMOBOLISMO CARREGADO}

Aristóteles, em seu "A Arte da Poesia" (5, 22, 1449 a 32), ressaltava que "a comédia é imitação de homens inferiores; não, todavia, quanto a toda a espécie de vícios, mas só quanto àquela parte do torpe que é ridículo". O ridliculo é apenas certo defeito, torpeza anódina e inocente (anódinon kai ou phthartikón, ou seja, kharan ablabé, um prazer não nocivo). Embora não sejam nocivas, as situaçōes risíveis oferecem oportunidades únicas de expressão de críticas que, de outra maneira, dificilmente poderiam ser expressas abertamente. "O que impede que se diga a verdade com uma risada?" (Ridentem dicere uerum quid uetat?, Hor. S. 1,1,24). "O humor $e$, com freqüência, mais forte e mais efetivo que a dureza para destrinchar grandes questōes" (ridiculum acri fortius et melius magnas plerumque secat res, Hor. S. 1,10,14-5). Através da zombaria é possível falar abertamente e, assim, questionar idéias e autoridades em voga. Portanto, a risada (rictus ou risus, Quint, 6,3), significa a possibilidade de expressão particularmente aberta da crftica, permitindo ao homem do povo o fustigamento das autoridades.

Como se poderia definir o que é risivel? Parece que a resposta pode ser encontrada no fato que a maioria, se năo todas (cf. Bergson, 1940, p. 95), as situações ridículas apresentam exageros. Justamente o exagero esiá na base da satira ou satura, um gênero especificamente latino (Quint. 10,1,93: satira quidem tota nostra est), ligada à lanx satura (prato cheio de frutas) e à satura (um tipo de salsicha) no sentido que a plenitude é a caracteristica comum destes diferentes e carregados ( $c$ f. a raiz "sa $=$ saturado) termos. Embora sem nenhuma relação direta, a caricatura também retira seu sentido da sobrecarga (caricare) que caracteriza as representaçōes ridículas. Ainda que o exagero não seja o único recurso cômico usado para "fazer o ouvinte mostrar os dentes na gargalhada", nas palavras de Horácio (S. 1,10,6), trata-se, contudo, do mais popular, graças a sua clareza (breuitas, Hor, S. 1,10,8). O exagero direto permite uma mais fácil compreensão, evitando longos processos de decodificação por parte do homem comum. Se isto é aplicável à sátira literária, segundo o conselho de Horácio $(S .1,10,8-9)$ que "você precisa da brevidade para permitir que o pensamento corra livremente sem se perder numa massa de palavras que pesará nos ouvidos", tanto mais o é em relação às inscriçōes vulgares parietais. $O$ exagero, na verdade, constitui caracteristicas comum mesmo nas mensagens verbais em Pompéia, como se torna claro na inscrição de Florônio (CIL IV 8767):

\section{Floronius, binet ac miles \\ leg, (ioni) uii hic, fuit, neque, mulieres scierunt, nisi paucae, et ses, erunt} (FIG. 1)

As antigas transcrições deste grafite de Della Corte (Della Cōrte, 1939; CIL IV 8767) e de Herescu (Herescu, 1969, p. 133) não conseguiam explicar, paleograficamente, as leituras que propunham de binet como benef (iciarius) (Della Corte), ou de ses, erunt como se de (de) runt (Della Corte) ou sederunt (CIL IV 8767 e Herescu, 1969, p. 126), em sentido obsceno (cf. Petrônio, Sat. 126,10; eqo etiam si ancilla sum, nunquam tamen nisi in equestribus sedeo). $A$ interpretação de Pisani (Pisani, 1973) permite explicar binet como bineta (cf. Luciano, Pseudolo- 
gista, 27) em sentido obsceno (= fututor) e, neste caso, pode-se interpretar a afirmaçáo como um exagero para provocar o riso: "Florônio, fodedor e soldado da sétima legião, esteve aqui (sc. uma taberna) e nenhuma mulher notou: eram apenas seis, pouco (sc. para tal garanhão)". O exagero podia, ainda, induzir ao riso pela zombaria, como neste caso (Della Corte, 1954, p. 329$, n. $851 \mathrm{~d}-\mathrm{m})$ :

[ded] uxisti octies, tibi superat ut habeas sedecies! Coponium fecisti; cretaria fecisti; salsamentaria fecisti; pistorium fecisti; agricola fuisti; aere minutaria fecisti; propola fuisti; luguncularia nunc facis, si cunnum barreira erigida entre 'alta' e 'baixa' arte não poderia ser mantida" (Wollen, 199, p. 72) e em oposiçăo, particularmente, à poesia concreta (Teles, 1977, p. 22; Crespo \& Bedate, 1963), ao poema máquina (Pignatari, 1965, p. 151), à poesia constelação (Gomringen, 1953) ou ao popcreto (Santiago, 1977, p. 46), os poemas figurativos da erudição antiga estavam completamente fora do campo de preocupação e percepção do homem comum. Mas, se é verdade que "o papel do simbolismo na vida quotidiana tendeu a ser negligenciado tanto por historiadores da cultura (preocupados com as "obras de arte") como por historiadores sociais (preocupados com a "realidade" social)" (Burke, 1989, p. 3) - e devemos acrescentar, ainda, os historiadores da literatura (Mac Donald, 1991, p. 238) - deve reconhecer-se que o simbolismo gráfico popular permanece quase completamente inexplorado cf. Gigante, 1979, p. 18). Infelizmente, estudos sobre práticas eruditas como pintura parietal (Rostovtzeff, 1919), desenho mural (White, 1957), quadros (tabulae, Perrin, 1989, p. 316), arte em geral (Dentzer, 1962) ou mesmo os estilos pompeianos (Schefold, 1972) e o imaginário da pintura antiga (Rouveret, 1989) são de diffcil uso quando tratamos dos rabiscos populares. Seria possivel, lendo apenas os anais do Parlamento, captar a mentalidade popular por detrás de um grafite como "não vote vomite"? (Melley, 1976, p. 104).

Para abordar a expressão estillstica gráfica devemos considerar três pontos. Em primeiro lugar, "estilo é poder... [e] criar estilo é criar uma ilusão de relações fixas e objetivas. O estilo envolve o acontecimento por linxseris, consummaris omnia.

Afirma-se que o anônimo foi garçom, oleiro, comerciante de peixe salgado, padeiro, agricultor, vendedor de quinquilharias de bronze, varejista $e$, agora, vendedor de garrafinhas. Para ter feito tudo, falta-lhe apenas um trabalho: a prática profissional do cunilingua (sobre o caráter usual desta prática, cf. Adams, 1987, p. 135; Johns, 1982, p. 141; contra Foucalt, 1986, pp. 23-4 e Veyne, 1986, p. 47).

A caricatura gráfica (Bergson, 1940, pp. 20-1) como uma forma especffica de arte, ainda que compartilhando algumas caracteristicas gerais do exagero da "baixa" arte, depende de esquemas simbólicos populares próprios, relativos ao desenho parietal e à representação humana, temas que abordo a seguir.

\section{O SIMBÓLICO E O ESTILISTICO NA ARTE GRÁFICA POMPEIANA}

Comparando poemas populares icônicos, com CIL IV 1595 (FIG. 2), 8031 (FIG. 3) ou 8329 (FIG. 4), com os poucos carmina figurata (Haerberlin, 1886) ou technopaegnia (Wendel, 1920, pp. 159-164) de autores eruditos como Teócrito (Wendel, 1920, pp. 159-164) e outros da Antologia Palatina, ficamos chocados pela dificuldade representada pelo uso de trocadilhos inusuais e obscuros (Willamovitz, 1899, p. 51). Diferentemente da arte pop moderna que reconheceu que "a interpretação mas fixa esta interpretaçăo como um acontecimento. Fornece o potencial para o controle do significado e, assim, do poder" (Hodder, 1990, p. 46). Significa, portanto, que o estilo torna-se poder através dos padrōes ou da repetição regular de traços significativos (Davis, 1990, p. 29). Este processo não é, necessariamente, um ato consciente, pois "o estilo pode ser, normalmente, passivo mas, mesmo assim, funciona iconicamente porque as pessoas reagem automaticamente de maneira simbólica e sem um estio aparente... portanto, pode dizer-se que mensagens éticas são muito mais frequentemente lidas do que de- 
liberadamente enviadas" (Sackkett, 1990, p. 37). Ainda mais, estilos não são apenas étnicos como sociais (Battisti, 1949, p. 42; Cândido, 1976, p. 169), diretamente relacionados à estratificação social (Lagopoulos, 1985, p. 266; Lagopoulos, inéd., p. 22). Embora evidências diretas das idéias populares estejam ausentes da tradição textual (Pollitt, 1989), podemos usar os dados disponiveis para reconstruir sua retórica gráfica (cf. Wallace-Hadrill, 1990, p. 147).

Como a caricatura trata, principalmente, da aparência humana (especialmente da face), devemos tentar definir como os romanos consideravam significativos os diferentes traços fisicos e como podem ser colocados, semioticamente, em um quadro de oposiçōes conceituais (QUADRO 1). As fontes escritas referem-se a diferentes sifnificados atribuidos ao cabelo, barba, sombrancelha, lábios, queixo, nariz, orelha e pescoço. A calvície (caluities) era associada à idade (Petr. Sat. 27; Suet. Galb. 20), e, assim, tanto a senilidade como à experiéncia e status decorrente do passar dos anos, enquanto que a negligência associava-se a muito cabelo, particularmente quando mal penteado (Ter. Heaut. 2,3,49; capillus passus, prolixus, circum caput reiectus negligenter). A barba era, normalmente, identificada com a adolescência (cf. Cic. N.D.1,30: quos aut imberbes aut bene barbatos uidetis), embora adultos barbados fossem ligados a imagens positivas, como os filosofos (Pers. 4,1) ou os romanos antigos (C. Coel. 14,33: barbula horrida ou barbicha tosca). Portanto, a calvíce e a barba produziam reações contraditórias, ressaltando a ambigüidade associada à faixa etária: juventude/barba/cabelo significam mais força flsica mas menos status e autoridade, velhice/face barbeada/calvicie associam-se a senilidade (tanto ffsica quanto mental) mas, por outro lado, também ao poder e autoridade advindos da passagem do tempo (QUADRO 2).

Sobrancelhas abundantes significavam alguém orgulhoso e arrogante até o ponto de serconsiderado duro e severo (Sen. Ep. 123,11); lábios finos geravam o riso (cf. Hier. Ep. 7,5: similem habent labra lactugam) (QUADRO 3); o queixo era associado ao poder (cf. Suet. Tib. 21). O nariz grande lembrava o sarcasmo (cf. Mart. 2,54,5: nil nasutius hac maligniusque; cf. Mart. 12,37,1), enquanto orelhinhas denotavam delicadeza e feminilidade (ou efeminação) (cf. Cic. Q. Fr. 2,15, a, 4), além de desatenção, em oposição à atençăo associada as orelhas grandes. O pescoço produzia sensaçőes contraditórias, sendo associado ao poder, liberdade e vida (Plaut. Trin. 2,4, 194) e, portanto, à sua privaçāo, cf. Prop. $2,10,85$ : dare colla triumpho). Estes traços físicos podem ser divididos considerando-se suas conotações em relação ao riso, ao poder e à autoridade como nos quadros 1,2 e 3 .

\section{AS CARICATURAS POMPEIANAS COMO UM SISTEMA SÓCIO-SEMIÓTICO}

Os estilos arquiteturais na pintura parietal pompeiana (Rostovtzeff, 1919, p. 150) eram, propriamente, um esquema decorativo interior (Wheeler, 1989, p. 12), ao mesmo tempo de caráter coletivo e de expressão privada (Perrin, 1989, p. 341), sendo a janela falsa a expressão mais perfeita deste tipo de ilusionismo consciente da classe alta (Rouveret, 1989, p. 299). A geração neroniana, embora muito breve, como todas as gerações (cf. Segal, 1991, p. 81), distinguia-se por traços particularmente marcados, em especial, pela cultura de libertos arrivistas (Stockton, 1990, p. 145; cf. Petr. Sat.) e pela introspecção das elites locais (Wilson, 1990, p. 379). Foi neste contexto que a caricatura desenvolveu-se nas paredes de Pompéia em oposição a estas expressōes da elite (cf. Petr. Sat. 29). Os rabiscos gráficos tinham uma própria Iógica interna (cf. Schefold, 1972, p. 251, sobre a lógica da pintura erudita), sujeita a estłmulos intrassistêmicos (Walicki, 1991, p. 101), estruturada, como a língua literária, em termos de compositio, iunctura e synthesis (Freudenburg, 1990, p. 197). Como este sistema nunca foi explicitado, devemos seguir o procedimento proposto por Hayek (Hayek, 1940: 530), ao tratar da análise de sistemas económicos, e chegar ao conhecimento a parte de elementos dispersos (cf. Blackburn, 1991, pp. 34-5). Auto-retratos imagines ridiculae permitem-nos notar como o desenho realça os atributos fisicos que podiam ser interpretado como ridiculos ou como sinais de falta de poder e autoridade, ou, ao contrário, como símbolos de status e prestigio. Comparemos três duplas de figuras (FIGS. 5, 6 e 7; QUADROS 4, 5 e 6). 
O mesmo esquema analítico pode ser aplicado a outros siufites (cf. CIL IV 1464; 7309; $7669 ; 7671 ; 8119 ; 8185 ; 10005 ; 10239)$. Notamos que auto-retratos tendem a ressaltar traços associados ao poder, autoridade, atenção, inteligência (cf. QUADRO 4), os traços amblguos sendo representados, provavelmente, pelo seu lado positivo (cf. QUADROS 5 e 6). A contrário, imagines ridiculae ressaltam conotaçōes reprováveis, provavelmente também determinantes nos traços amblguos. Podemos concluir, portanto, que, embora não explicitamente, havia uma escolha definida de atributos informando uma estrutura icônica carregada de significado (TABELAS 1, 2 e 3). Deve notar-se que a composição sintática depende de conotaçōes exossemióticas ou culturais, ou seja, de uma associação arbitrária de traços físicos a significados comportamentais implicitos. Ainda mais, estes desenhos demonstram que o homem comum não apenas criticava autoridades (cf. CIL IV, 9226 = FIG. 6), como, também, usava sua própria criatividade estilistica e simbólica para levar a cabo esta crítica. Assim, não podemos "superestimar o poder das formulações ideológicas para controlar e manipular as pessoas e subestimar a habilidade das classes baixas em discernir as ideologias pelas quais as elites tentavam dominá-los" (Trigger, 1989, p. 786; cf. Rowlands, 1983, p. 111).

A caricatura gráfica permite-nos, ainda, compreender como o ethos popular era profundamente afetado por contradiçōes graças, principalmente, à desumanizaçāo derivada da escravidão como instituição social. A escravidão estava no centro da exploração do homem e o fato que "todos os homens são livres ou escravos" (omnes homines aut liberi sunt aut serui), nas palavras de Gaio $(1,3,9)$, significava que um processo de despossessão humana atingia a própria gente simples. A diferença dos jogos de gladiadores representados na pintura erudita (PI. N.H. 35,51-2), os grafites expressam os sentimentos dos torcedores comuns por seus heróis (Funari, 1989, pp. 40-42; 63-66). Enquanto na elite havia divergências sobre o bom gosto dos munera (Ville, 1981), estes espetáculos gladiatórios estavam no centro da percepção popular da vida. Gladiadores livres ou escravos provavam que homens podiam ser condenados à morte (cf. Sen. Ep. 87: comparare homines ad gladium) para prazer da massa. Em última instância, a sujeição privada de homens a homens, como escravos e senhores, justificava-se, na mentalidade popular, pela posse coletiva de homens (os gladiadores) a serem sacrificados para entretenimento popular. $O$ trabalho e a morte dos gladiadores estavam para o povo como o trabalho e a morte dos escravos privados estavam para seus senhores. Este processo de despossesão do homem de sua humanidade apresenta-se muito claro nos rabiscos dos torcedores (cf. CIL IV 8055-6; 10221; 10236). Dois exemplos bastarão para distinguir diferentes nfveis de representação dos traços humanos de acordo com a valorização social do homem representado (CIL IV 8017; 10237) (FIGS. 8 e 9).

Em CIL IV 8017 (FIG. 8), homem e animal estão representados como seres iguais, como realmente eram na uenatio enquanto espetáculo público de gala (apparatus, Cic. Off. 2,16,55), estando o rosto de Venustus, simplesmente, não representado. A maioria dos traços usados no desenho de Venustus referem-se a armas e roupas ligadas à luta: nada mais é que um lutador sem face para a satisfação do povo (cf. FIG. 9 e TABELA 4). Em CIL IV 10237, nota-se facilmente que os atributos tornam-se crescentemente humanos dos gladiadores aos flautistas até os dois deuses visíveis acima da representação (FIG. 9 e TABELA 5). Devemos concluir, portanto, que a percepção da posição social expressava-se, inconscientemente, através de um sistema gráfico semiótico e que, desta maneira, contribuia para o reforço dos laços sociais de exploração.

A caricatura, em Pompéia, podia adquirir um caráter muito abstrato, algumas vezes captando, em um mesmo quadro, três niveis interrelacionados, verbal, fónico e icónico. Constitui um bom exemplo a epigrafe CIL IV 8329 (FIG. 10), ao mesmo tempo uma mensagem escrita (Sseuera phelassss = seuera felas = Severa, chupas), uma expressão fônica do ato, graças å repetiāo da letra s, e uma caricatura dos parceiros (Fig. . ). O alto grau de abstração demonstra que a aisthesis e a expressão populares, antes que simples, cruas e diretas, podiam atingir altos niveis sistêmicos de complexidade e subjetividade (Funari, 1987). 


\title{
6. EM DIREÇÃO A ESPECIFICIDADE DO ETHOS POPULAR
}

Podemos concluir este estudo dos desenhos parietais gráficos ressaltando três aspectos interrelacionados:

1. Havia um sistema semiótico especlfico relativo à expressão parietal gráfica;

2. Este sistema era, a um só tempo, simbólico e social. Era simbólico na medida em que cresceu em oposição à pintura erudita como uma técnica de rabisco a céu aberto com regras de composição próprias. Era, também, social pois este sistema simbólico expressava, através das contradições sociais, os sentimentos populares. Particularmente claras apresentam-se as diferenças de classe e status como definidoras tanto da percepção como fruição populares;

3. Como consequência, através de mecanismos simbólicos autônomos, a caricatura servia, ao mesmo tempo, para criticar autoridades (cf. CIL IV 9226), para reforçar a diferenciação e exploração social (cf. CIL 10237) e para expressar a auto-estima (cf. CIL IV 9008), interesses (cf. CIL IV 8017) e paixōes (cf. CIL IV 8329) populares.

Mas, talvez, as caricaturas antigas devam ser, simplesmente, admiradas por sua beleza e espontaneidade. Se assim for, terminaria lembrando as palavras de Machado de Assis, muito a propósito, sobre as expressōes populares:

"Notai que o que legitima um um vocábulo destes, é a sua expontaneidade. Eles nascem como as plantas da terra. Não são flores artificiais de academias, pétalas de papelāo recortadas em gabinetes, nas quais o povo não pega. Ao contrário, as geradas naturalmente e que acabam entrando nas academias".

(A Semana, 1893).

\section{AGRADECIMENTOS}

Expresso meu reconhecimento aos seguintes amigos e colegas que me forneceram artigos (alguns inéditos), trocaram idéias e me ajudaram de diferentes maneiras: Peter Burke, lan Hodder, Alexandros-Phaidon Lagoulos, Michael Rowlands, Bruce G. Trigger e Ellen Meiksins Wood. A responsabilidade pelas idéias expostas refere-se apenas ao autor.

\begin{abstract}
Studying what is popular and satyrical, the paper looks for a difinition of ordinary people's ethos at Pompeii. The analysis of popular culture in Ancient Society is carried out through the characterization of Satyre and Caricature as popular expressions. The stylistic symbolism shown in wall drawing is studied in opposition to learned expressions (carmina figurata). The socio-semiotic system relating to caricature is studied thanks to Pompeian evidence. The paper ends with a difinition of ordinary people's ethos.
\end{abstract}




\section{REFERËNCIAS BIBLIOGRÁFICAS}

ADAMS, J.N. The Latin Sexual Vocabulary. Londres: Duckworth, 1987.

BATTISTI, C. Avviamento allo studio del latino volgare. Bari: Leonardo da Vinci editrice, 1949.

BAKHTINE M. L' oeuvre de Francois Rabelais et la culture populaire du Moyen-Age et sous la Renaissance. Paris: Gallimard, 1970.

BERGSON, H. Le rire. Essai sur la signification du comique. Paris: PUF, 1940.

BIANCHI-BANDINELLI, R. Archeologia e cultura. Milão: Ricciardi, 1961.

- Rome, the centre of the power. Roman art to AD 200. Londres: Thames \& Hudson, 1970.

-. Del Helenismo a la Edad Media. Madri: Akal, 1981.

BLACKBURN, R. Fin de siecle: socialism after the crash, New Left Review, 185, p. 5-67, 1991.

BRENDEL, O. J. Prolegomena to the study of Roman Art. New Haven: Yale University Press, 1979.

BROWNE, R. B. Redefining literature, Journal of Popular Culture, 23,3, p. 11-21, 1989.

BRUNT, P. A. Schiavi e classi subalterne nella comunità romano-italica, in: AA.VV. Storia della società italiana, 2, Milão, Teti, 1983, p. 95-132.

BULFORD A. Craftsmen in Greek and Roman Society. Londres: Duckkworth, 1972.

BURKE, P. The historical anthropology of early modern Italy. Essays on perception and communication. Cambridge: CUP, 1989a.

-. A cultura popular na Idade Moderna. São Paulo: Cia das Letras, $1989 \mathrm{~b}$.

CANDIDO, A. Literatura e Sociedade. São Paulo: Cia Ed. Nacional, 1976.

CEBE, J. P. La caricature et la parodie dans le monde romain antique des origines à Juvenal. Paris: de Boccard, 1966.

CRESPO, A. \& BEDATE P. G. Situación de la poesia concreta. Revista de Cultura Brasileña, 5, P. 15-35, 1963.

CROCE, B. Poesia, storia. Pagine tratta da tutte le opere a cura dell'autore. Nápoles: Ricciardi, s.d.

DAVIS, W. Style and history in art history, In: CONKEY M. \& HASTORF, C. (eds), The uses of style in Archaeology, Cambridge: CUP, 1990, p. 18-31.

DELLA CORTE, M. Iscrizioni a Pompeii, Notizie degli scavi, p. 293, 1939.

-_. Case ed abitanti di Pompeii. Roma: L'Erma, 1954.

DENTZER, J. M. La tombe de C. Vestoirius dans la tradition de la Peinture italique, Mélanges de l'Ecole Francaise de Rome, 74, p. 533-594, 1962.

DE MARTINO, F. Nuovi studi di economia e diritto romano. Roma: Riuniti, 1988.

DIAZ, M. E. The satiric penny pen press for workers in Mexico, 1900-1910: a case study in the politicisation of popular culture. Journal of Latin American Studies, 22,3, p. 497-526, 1990.

FOUCAULT, M. The care of the self. The history of sexuality, volume three. Harmondsworth: Penguin, 1986.

FREUDENBURG, K. Horace's satiric program and the language of contemporary theory in Satires, 2,1, American Journal of Philology, 111,2, p. 187-203, 1990.

FUNARI, P.P. A. Cultura(s) dominante(s) e cultura(s) subalterna(s) em Pompéia: da vertical da cidade ao horizonte do possivel, Revista Brasileira de Historia, 7, p. 33-48, 1987.

- Cultura popular na Antigüidade Clássica. São Paulo: Contexto.

GICHON, M. Who were the enemies of Rome on the Limes Palestinae, Studien zu den Militaergrenzen Roms III, Aalen: 13. Internationaler Lemeskongress, 1983, p. 584-592.

GIGANTE, M. Civiltả delle forme letterarie nell'antica Pompeii. Nápoles: Bibliopoli, 1979. 
GOMRINGEN, E. Konstellationen. Frankfurt: 1953.

GOLBY, J. M. \& PURDUE, A. W. The civilization of the crowd. Popular cultures in England 1750-1900. Londres: Batsford, 1984.

GUILLEMAN, Compte-rendu, Révue des Etudes Latines, 13, p. 404-6, 1935.

GUINSBURG, C. An interview to Keith Luria \& ROMULO Gandolfo, Radical History Review, 39, p. 89-111, 1986.

HAHN, I. Klassengebundheit, Tendenz und Anspruch auf Objektivitaet der Antiken Geschichtsschreibung, in: Alonso-NUNES J. M. (ed), Geschichtsbild und Geschichtsdenken im Altertum, Darmstad: W. B., 1991, p. 363-405.

HAEBERLIN, C. Carmina Figurata Graeca. Hanover: Janeck, 1886.

HARRIS, W. V. On the applicability of the concept of class in Roman History, in: Forms of Control and Subordination in Antiquitity, Tóquio: p. 598-610, 1988.

HAYEK, F. A. von The uses of knowledge, American Economic Review, p. 525-540, 1940.

HERESCU, N. J. Sur le sens érotique de sedere, Glotta, 38, p. 125-134, 1969.

HILL, C. History and Present. Londres: South Place Ethical Society, 1989.

HOBSON, D. W. House and household in Roman Egypt, Yale Classical Studies, 28, p. 211-229, 1985.

HODDER, I. Style as historical quality in art history, in: CONKEY, M. \& HASTORF, C., (eds). The uses of style in Archaeology, Cambridge: CUP, p. 44-51, 1990.

HOGGART, R. The uses of literacy. Harmondsworth: Penguin, 1986.

HOWKIKNS, A. Labour history and the rural poor. 1850-1980, Rural history, economy, society, culture, 1,1, p. 113-122, 1990.

JOHN, C. Sex or symbol? Erotic images os Greece and Rome. Londres: British Museum Publications, 1982.

LAGOPOULOS, A. P. Historical materialism, semiotics and urban space: towards a social semiotics of urban texts, Ars Semeiotica, 8,3/4, p. 253-268, 1985.

- Sign conceptions in architecture and the fine arts in ancient Greece and Rome. Tessalonica: manuscrito inédito.

LISSBERGER, E. Das Fortleben der roemischen Elegiker in den Carmina epigraphica. Tuebingen: E. Goobelin, 1934.

MACDONALD, A. H. Theme and style in Roman historiography, in: ALONSO-NUNEZ, J. M. (ed)., Geschichtsbild und Geschichtsdenken im Altertum, Darmstadt: W. B., p. 220-238, 1991.

MACMULLEN, R. Roman social relations, $50 B C$ to $A D$ 284, New Haven: Yale University Press, 1974.

-Changes in the Roman Empire. New Jersey: Princeton University Press, 1990.

MATTEWS, T. Roman life and society, in: GRIFFIN, J. \& MURRAY, O. (eds), The Roman WorL, Oxtord: OUP, p. 338-360, 1990.

MELLY, G. The writing on the wall. Londres: Elmtree books, 1976.

NCOLET, C. Renche a Cesar. Economie et societe dans la Rome Antique. Paris: Gallimard. 1988.

ORSTED, P. Aomen imperial economy and Romanization. Copenhagen: Mueseum Tusculanum Pross, 1905.

PEARAN, Y. Peinun et societs a Rome: question de sociologie, sociologie de rart, sociologie de la perception, Mctinges Pierre Lóveque, 3, Paris: Belles Lettres, p. 313-342, 1989.

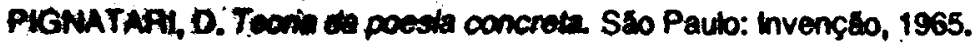

ProAN1, V. Su un graitio pompeiano, Parala del Passato, 28, p. 213-5, 1973. 
POLLITT, J.J. The art of Rome c. 753 BC-AD 333, Sources and documents. Cambridge: CUP, 1989.

RODENWALDT, G. The transition to late-classical art, in: Cambridge Ancient History, Cambridge: CUP, p. 545-565, 1939.

ROSENBERG, B. Was there a popular culture in the Middle Ages? Journal of Popular Culture, 14,1, p. 149-154, 1980.

ROSTOVTZEFF, M. Die hellenistisch-roemische Architekturlanschaft, Mitteilungen des Deutschen Archaeologischen Institut, Roemische Abteiling, 26, p. 1185-1911.

-. Ancient decorative wall-painting, Journal of Hellenic Studies, 39, p. 144-163, 1919.

ROUVERET, A. Histoire et imaginaire de la peinture ancienne ( $V$ s. av. J.C. -1 s. ap. J.C.). Roma: École Francaise de Rome, 1989.

ROWLANDS, M. Objectivity and subjectivity in Archaeclogy in: Spriggs, M. (ed), Marxist perspectives in archaeology, Londres: p. 108-113, 1983.

SACKETT, J. R. Style and ethnicity in archabology: the case for isochretism, in: CONKEY, M. \& HASTORF, C. (eds). The uses of style in Archaelogy, Cambridge: CUP, p. 32-43, 1990.

SANTIAGO, S. Paulistas e mineiros, Revista de Cultura Vozes, 1, 71, p. 39-46, 1977.

SCHEFOLD, K. La peinture pompéienne. Essai sur l'evolution de sa signification. Bruxelas: Latomus, 1972.

SCOBIE, A. Slums, sanitation and mortality in the Roman World, Klio, 68, p. 399-433, 1986.

SEBILLOT, P. Le Folklore. Paris: Garnier, 1913.

SEGAL, L. Socialism, Feminism and Future, New Left Review, 185, p. 81-91, 1991.

STOCKTON, D. The founding of the Empire, in: BORDMAN, J., GRIFFIN, J. MURRAY, O. (eds), The Roman World, Oxford: OUP, 121-149, 1990.

TELES, G.M. O nome da poesia concreta, Revista de Cultura. Vozes, 1, 71, p. 19-22, 1977.

TRIGGER, B.G. Hyperrelativism, responsability, and the social sciences, Canadian Review of Sociology and Anthropology, 26,5, p. 776-797, 1989.

VEYNE, P. A homossexualidade em Roma, In: ARIÉS P. \& BÉJIN, A. (eds), Sexualidades ocidentais, São Paulo: Brasiliense, p. 39-49, 1986.

VILLE, G. La gladiature en Occident des origines à la mort de Domitien. Paris: De Boccard, 1981.

WALICKI, A. From Stalinism to post-communist pluralism: the case of Pland, New Left Review, 185, p. 93-121, 1991.

WALLACE-HADRILL, A. Roman arches and Greek honours: the language of power at Rome, Procedings of the Cambridge Philological Society, 216, p. 143-181, 1990.

WENDEL, C. Ueberlieferung und Entstehung der Theocritsscholien. Berlim: Weidmannische, 1920.

WHEELER, M. Roman art and architecture. Londres: Thames \& Hudson, 1989.

WHITE, J. Perspective in ancient drawing and painting. Londres: S. Press of Hall Studies, 1957.

WILAMOWITZ, U.V. Die Greschischen Technopaegnia, Jahrbuch des Deutschen Archaeologischen institut, 14, p. 51-59, 1989.

WILSON, R. J. A. Roman Art and Architecture, in: BORDMAN, J., GRIFFIN, T., MURRAY, O. (eds), The Roman World, Oxford: OUP, p. 361-400, 1990.

WOOD, E. M. Peasant-citizen and slave. The foundations of Athenian Democracy. Londres: Verso, 1989.

WOOLEN, P. Scenes from the future: Komar \& Melamid, New Left Review, 185, p. 68-80, 1991. 


\begin{tabular}{|c|c|c|}
\hline \multicolumn{3}{|c|}{ QUADRO 1} \\
\hline APARÊNCIA FÍSICA & ADJETIVOS & CONOTAÇÃO \\
\hline $\begin{array}{l}\text { CALVÍTIES - } \\
\text { (CALVÍCIE) }\end{array}$ & $\begin{array}{l}\text { CALVVS } \\
\text { (CALVO) }\end{array}$ & $\begin{array}{l}\text { - SENECTUS } \\
\text { (SENILIDADE) }\end{array}$ \\
\hline $\begin{array}{l}\text { CAPILLVS } \\
\text { (CABELO) }\end{array}$ & $\begin{array}{l}\text { PASSVS } \\
\text { PROLIVS } \\
\text { REIECTVS }\end{array}$ & $\begin{array}{l}\text { NEGLIGENTIA } \\
\text { (NEGLIGÊNCIA) }\end{array}$ \\
\hline $\begin{array}{l}\text { BARBA } \\
\text { (BARBA) }\end{array}$ & $\begin{array}{l}\text { (SE JOVEM) } \\
\text { (SE ADULTO) }\end{array}$ & $\begin{array}{l}\text { JUVENTUDE } \\
\text { SABEDORIA }\end{array}$ \\
\hline $\begin{array}{l}\text { SVPERCILIVM } \\
\text { (SUPERCILIO) }\end{array}$ & SVPERCILIOSVS & - PETULÂNCIA \\
\hline $\begin{array}{l}\text { LABRA } \\
\text { (LÁBIOS) }\end{array}$ & $\begin{array}{l}\text { SIMILEM LACTUGAM } \\
\text { (COMO ALFACE) }\end{array}$ & RISIVEL \\
\hline \multicolumn{3}{|l|}{$\begin{array}{l}\text { MAXILLA } \\
\text { (QUEIXO) }\end{array}$} \\
\hline $\begin{array}{l}\text { NASVS } \\
\text { (NARIZ) }\end{array}$ & NASVTVS & SARCASMO \\
\hline $\begin{array}{l}\text { AVRIS } \\
\text { (ORELHA) }\end{array}$ & $\begin{array}{l}\text { AVRICVLA } \\
\text { AVRITVS }\end{array}$ & $\begin{array}{l}\rightarrow \text { FEMINILIDADE } \\
\rightarrow \text { ATENÇÁO }\end{array}$ \\
\hline
\end{tabular}




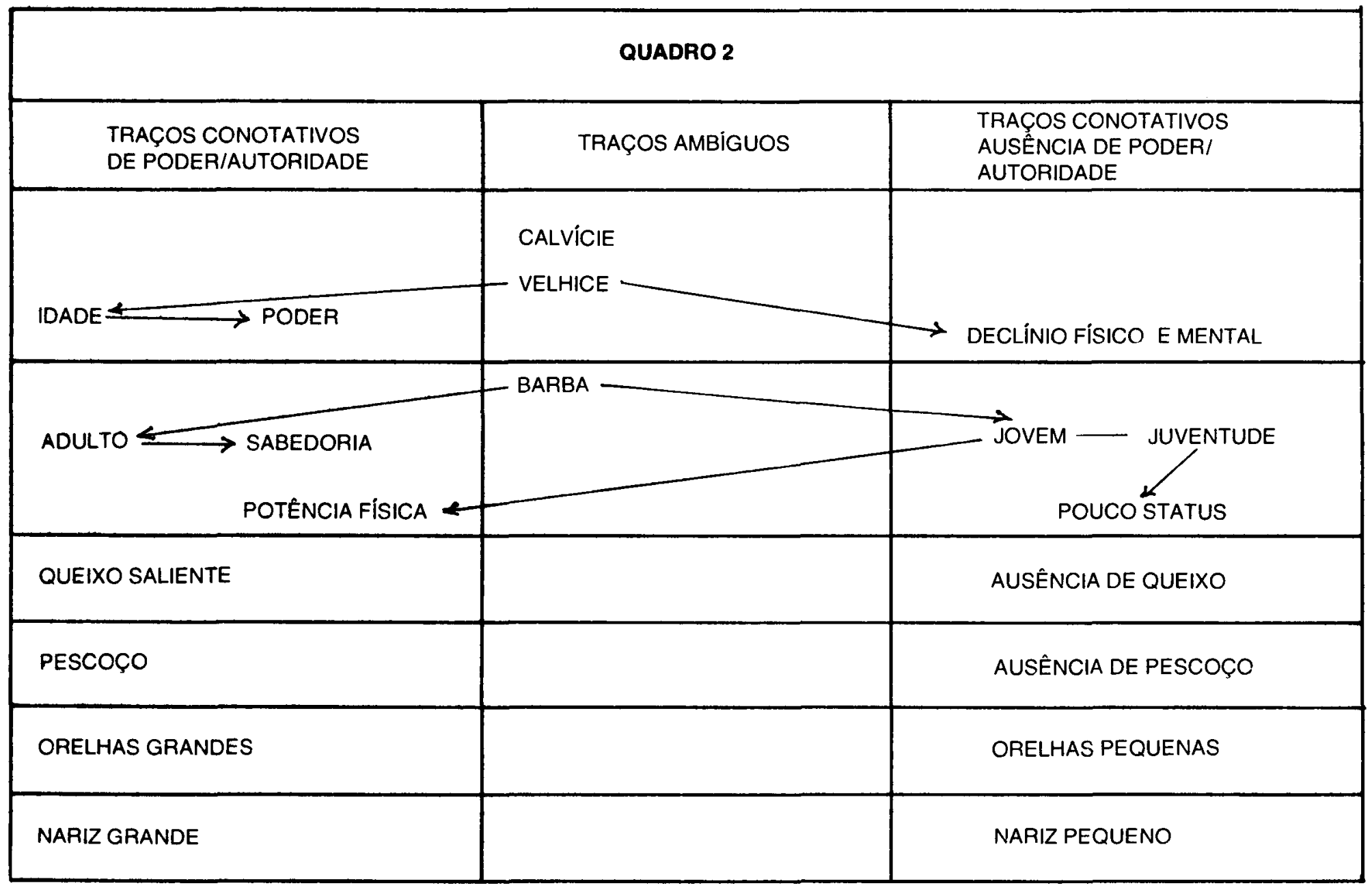




\begin{tabular}{|c|c|}
\hline \multicolumn{2}{|c|}{ QUADRO 3 } \\
\hline TRAÇOS RISIVEIS & \\
\hline CARACTERISTICA FISICA CONOTAÇÃO \\
\hline CAPILLVS PASSUS & \\
\hline PROLIXVS $\longrightarrow$ NEGLIGENTIA (NEGLIGÊNCIA) & $\longrightarrow$ PETULÂNCIA \\
\hline REIECTVS $\longrightarrow$ RIDICULO \\
\hline SVPERCILIOSVS $\longrightarrow$ SARCÁSTICO \\
\hline LABRA (SIMILEM LACTUGAM) & \\
\hline NASVTVS & \\
\hline AVRICVLA & \\
\hline
\end{tabular}




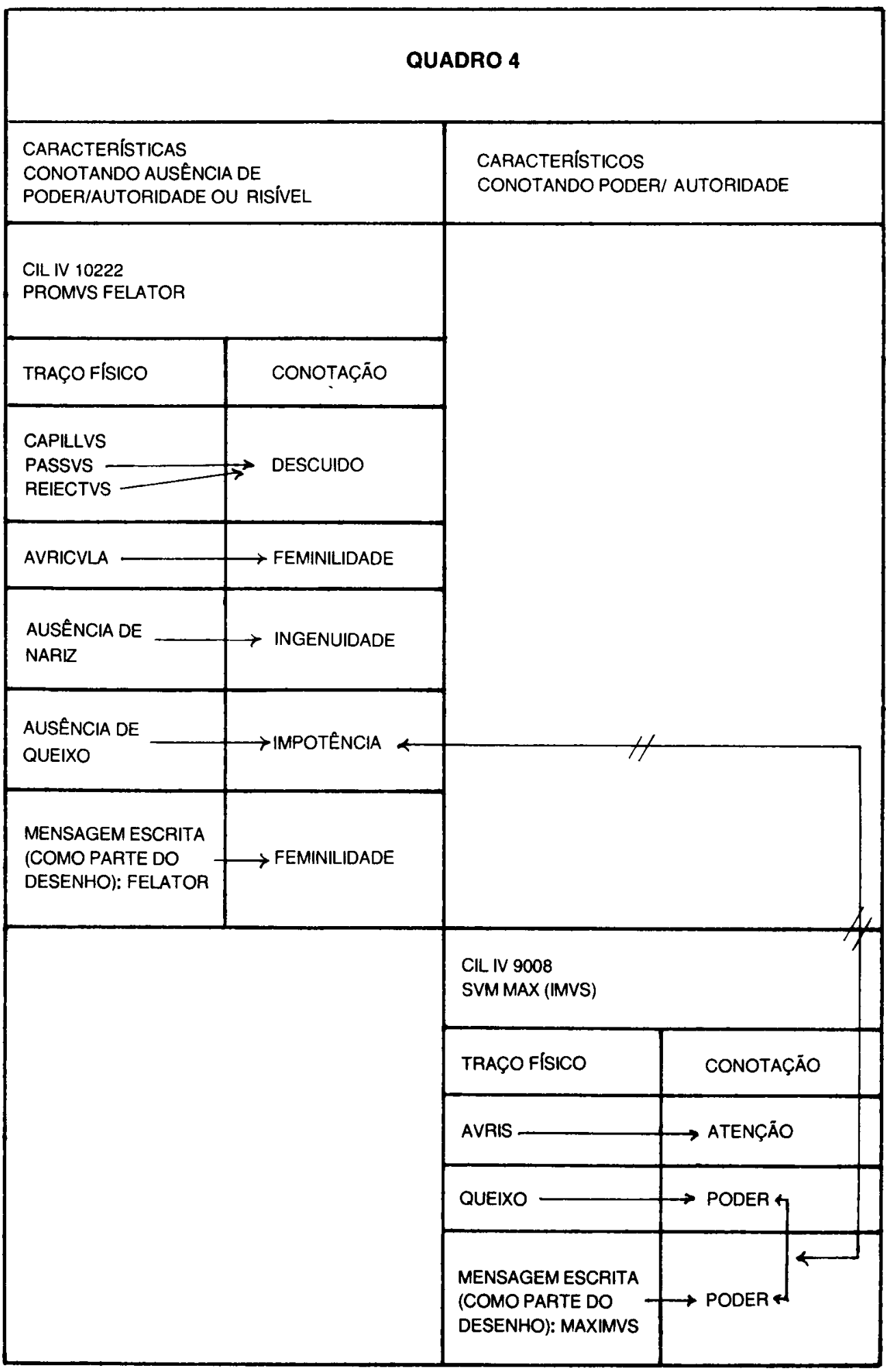




\section{QUADRO 5}

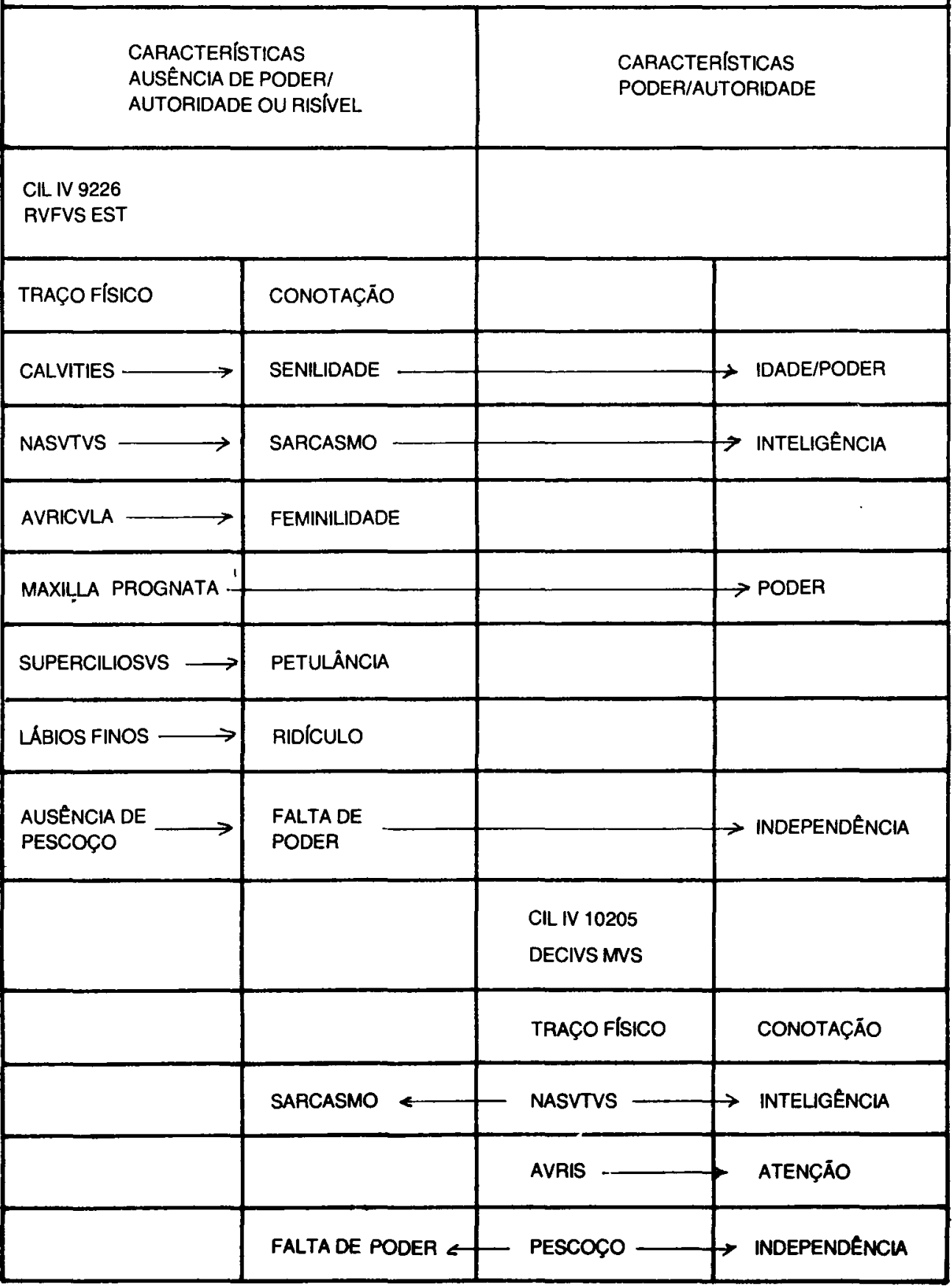




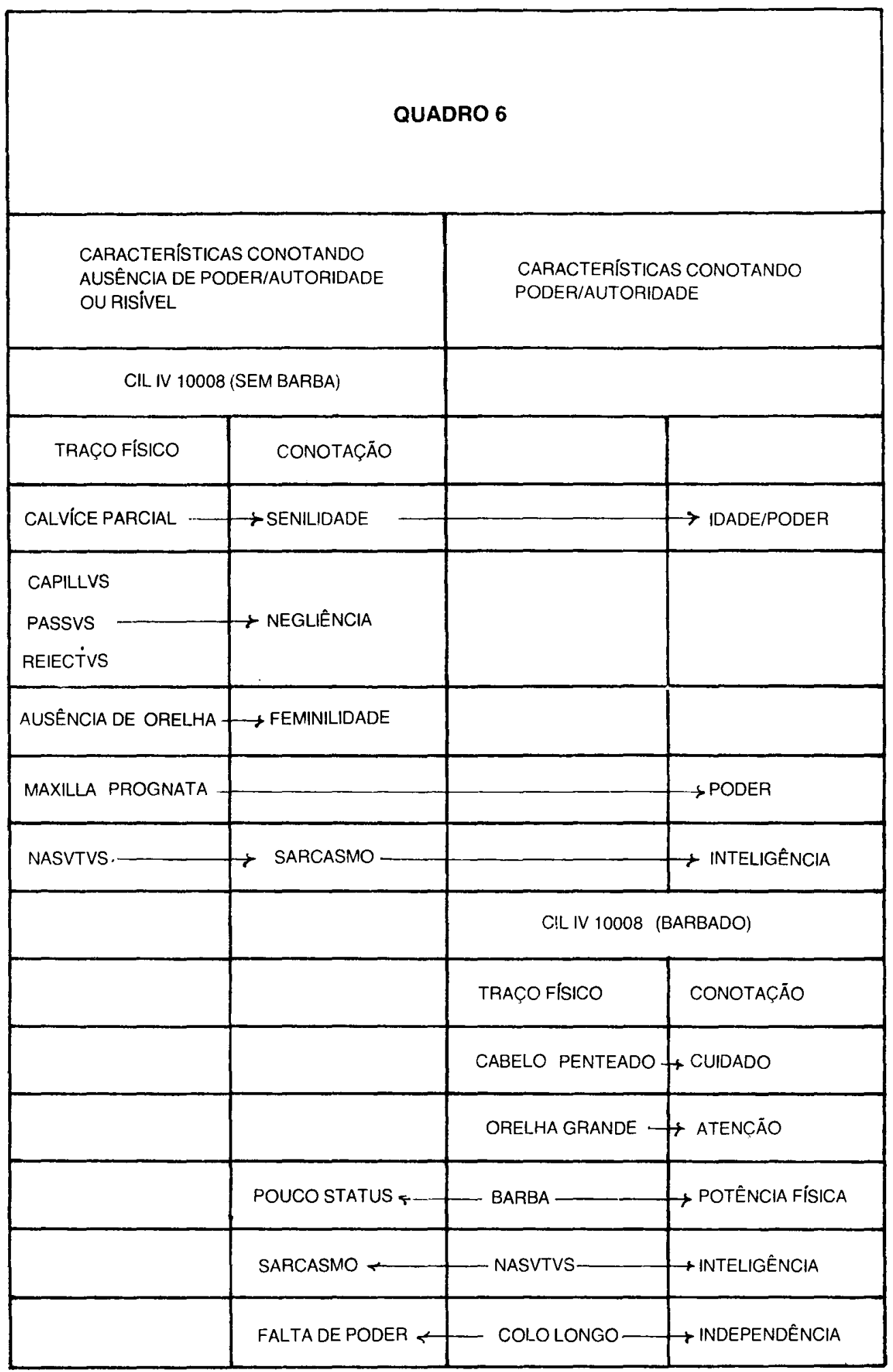




\section{TABELA 1}

CARACTERISTICAS DO QUADRO 4:

\begin{tabular}{|c|c|c|}
\hline POSITIVAS: & 0 & 3 \\
\hline NEGATIVAS: & 5 & 0 \\
\hline AMBIGUAS: & 0 & 0 \\
\hline & CIL IV 10222 & CIL IV 9008 \\
\hline
\end{tabular}

TABELA 2

CARACTERÍSTICAS DO QUADRO 5:

\begin{tabular}{|c|c|c|}
\hline POSITIVAS: & 1 & 1 \\
\hline NEGATIVAS: & 3 & 0 \\
\hline AMBIGUAS: & 3 & 2 \\
\hline & CIL IV 9226 & CIL IV 10205 \\
\hline
\end{tabular}

TABELA 3

CA.RACTERISTICA DO QUADRO 6:

\begin{tabular}{|c|c|c|}
\hline POSITIVAS: & 1 & 3 \\
\hline NEGATIVAS: & 2 & 0 \\
\hline AMBIGUAS: & 2 & 2 \\
\hline & CIL IV 10008 (SEM BARBA) & CIL IV 10008 (BARBADO) \\
\hline
\end{tabular}




\section{TAEELA 4}

TRAÇOS DA FIGURA 8 (VENVSTVS):

\begin{tabular}{|l|c|c|}
\hline CARACT. DO CORPO HUMANO: & 7 TRAÇOS & $21,2 \%$ \\
\hline TRAÇOS AMBIGUOS (PERNAS): & 2 TRAÇOS & $6 \%$ \\
\hline TRAÇOS LIGADOS À LUTA: & 24 TRAÇOS & $72,7 \%$ \\
\hline
\end{tabular}

\section{TABELA 5}

TRAÇCS DA FIGURA 9 (TRAÇOS DA FACE POR FIGURA HUMANA):

\begin{tabular}{|c|c|c|}
\hline GLADIADORES & 3.5 & $(100)$ \\
\hline FLAUTISTAS & 7 & $(200)$ \\
\hline DEUSES & 20 & $(571)$ \\
\hline
\end{tabular}

FIG. 8 OS POUCOS TRAÇOS, ESSENCIAIS PARA A CARACTERIZACCÃO DE VENVSTVS COMO UM LUTADOR, NĀO APRESENTAM NENHUMA CARACATERÍSTICA HUMANA (COMO EXPRESSÕES FACIAIS) E, DESTA FORMA, SEU DESENHO ASSEMELHA-SE MUITO À REPRESENTAÇÃO. POIS OS DOIS DESENHOS ABAIXO NÃO SÃO REPRESENTAÇÕES CLARAS DE IDOLOS. 
FLORONN/-

bINES DS:WLEI

SGA- Tाr HIL.

rut. NGave

SCIE LiE

SCIERUNTINII

CAY CA SET
SFSER
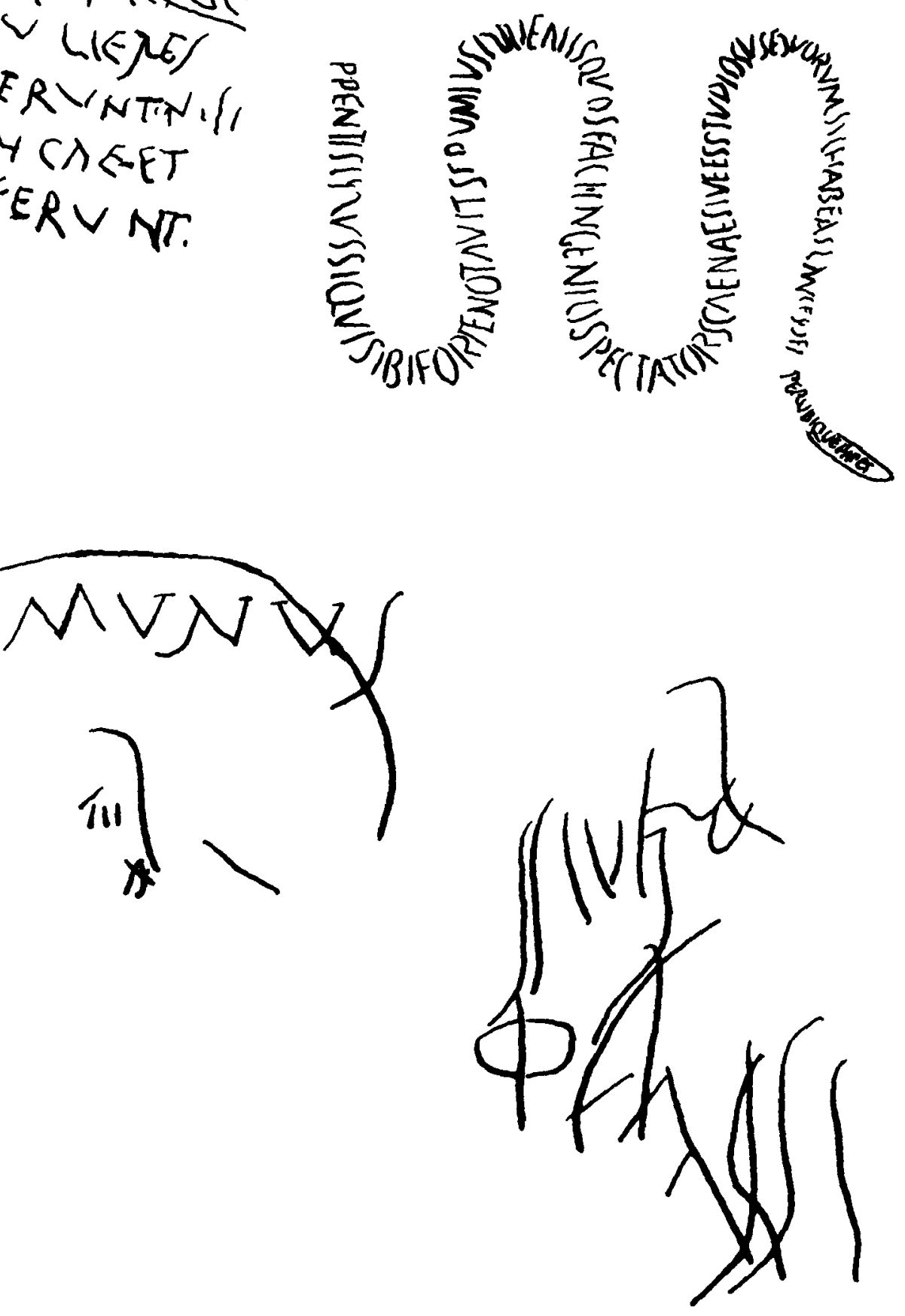

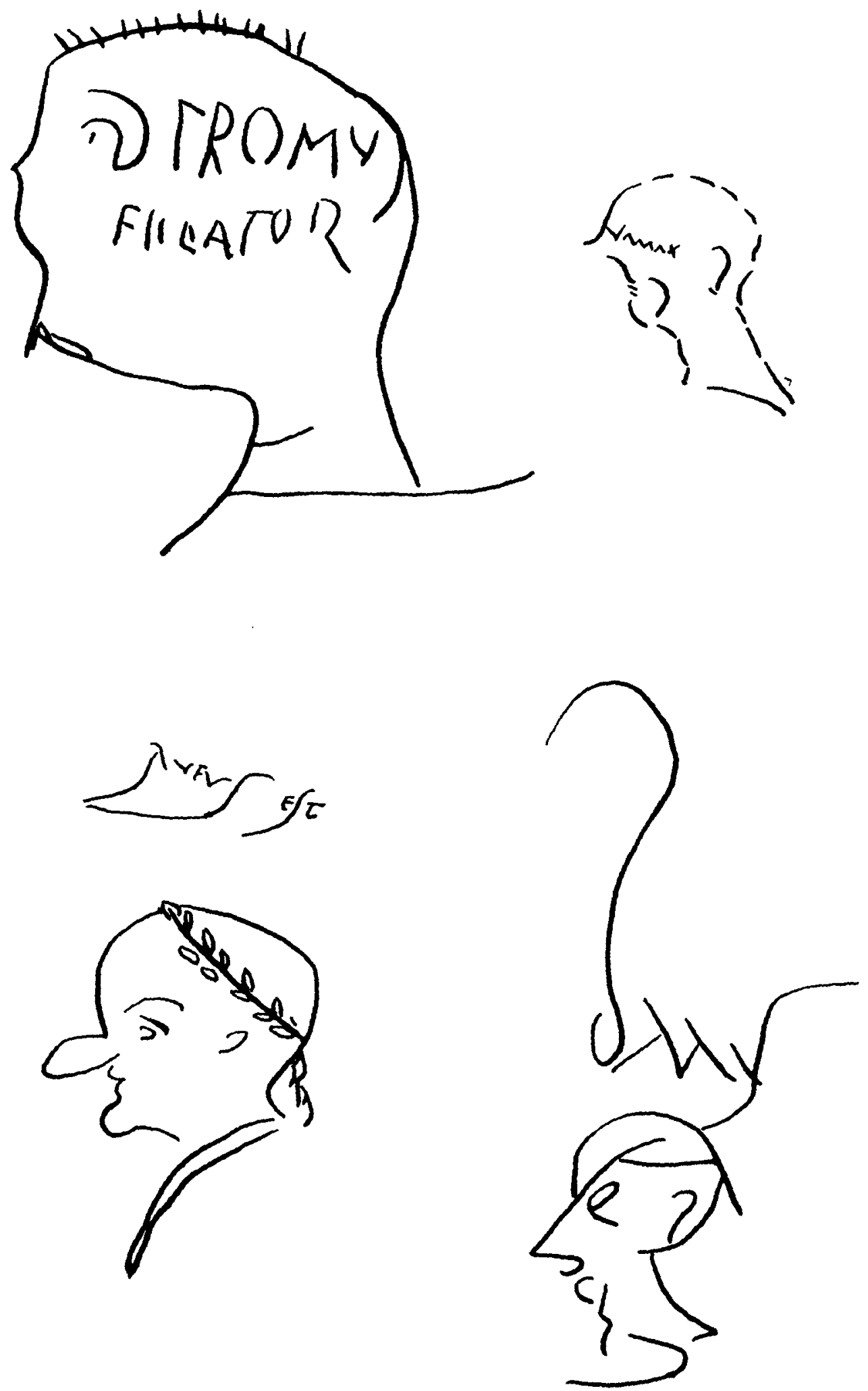
137

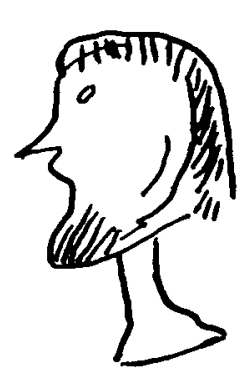

$$
5^{4}
$$

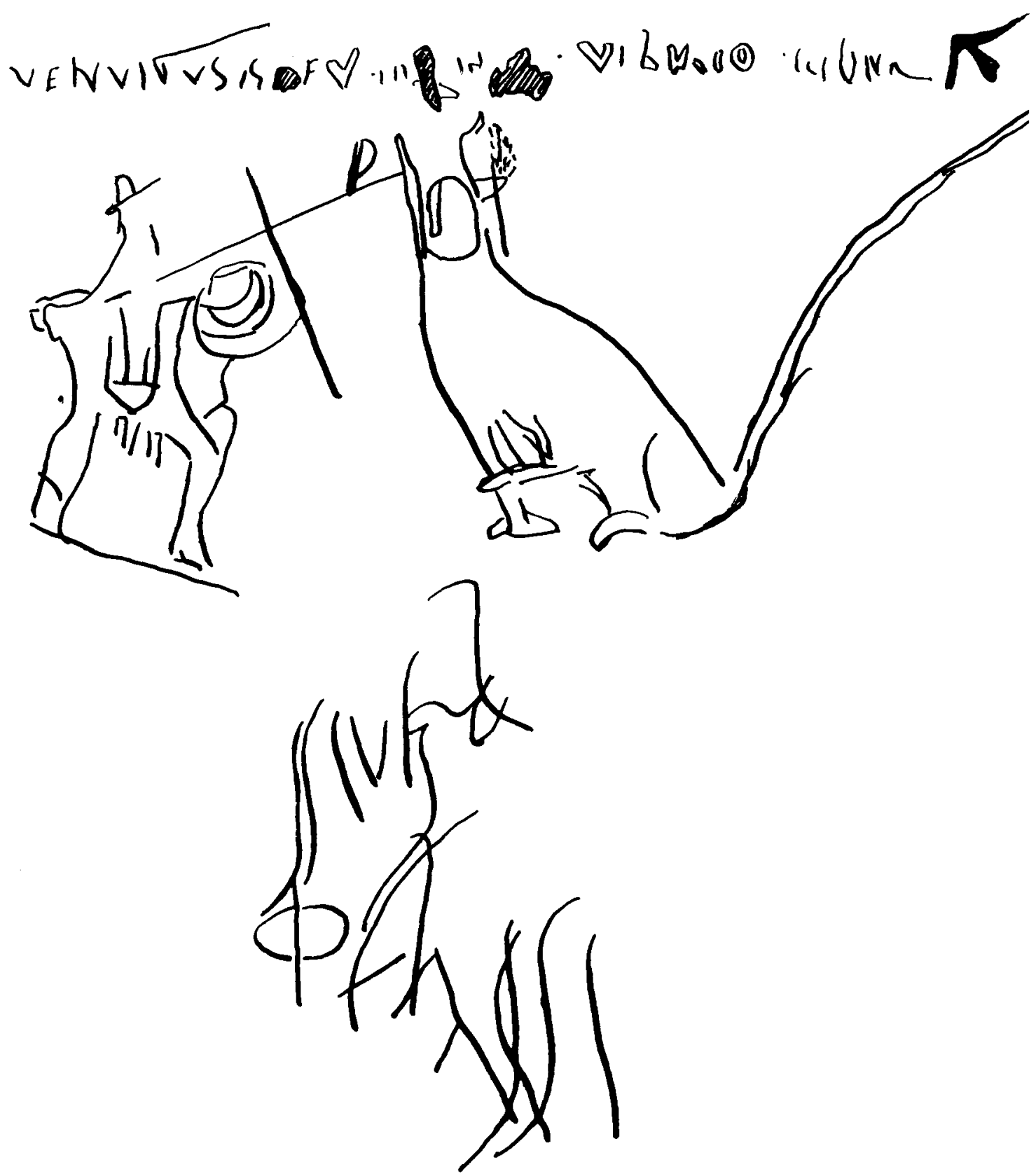




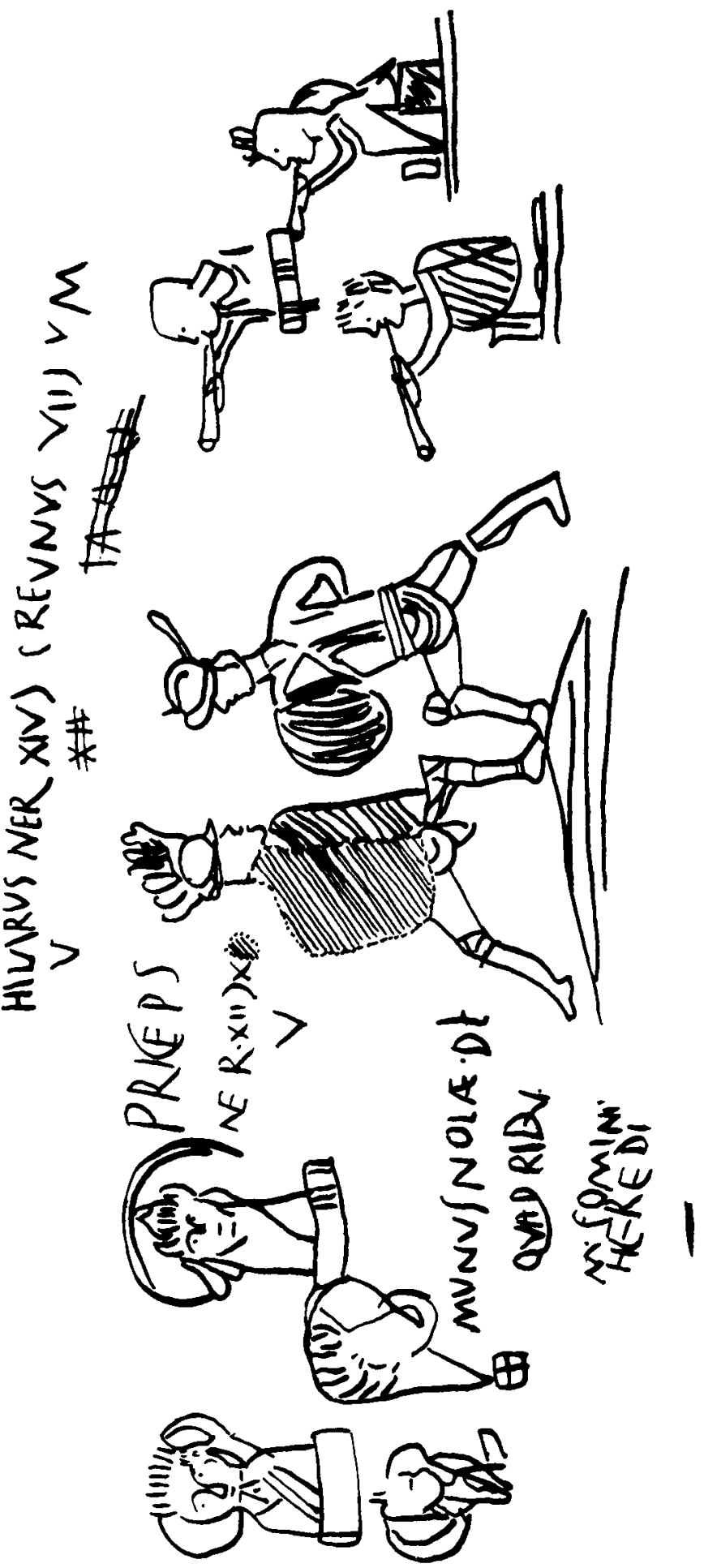

